\title{
Active Contouring Based on Gradient Vector Interaction and Constrained Level Set Diffusion
}

\author{
Xianghua Xie, Member, IEEE
}

\begin{abstract}
This paper presents an extension of our recently introduced MAC model to deal with the initialization dependency problem that commonly appears in edge-based approaches. Its dynamic force field, unique bidirectionality, and constrained diffusion-based level set evolution provide great freedom in contour initialization and show significant improvements in initialization independency compared to other edge-based techniques. It can handle more sophisticated topological changes than splitting and merging. It provides new potentials for edge-based active contour methods, particularly when detecting and localizing objects with unknown location, geometry, and topology.
\end{abstract}

Index Terms-Active contour, edge based, initialization flexibility, level set method, object segmentation.

\section{INTRODUCTION}

D EPENDING on the assumption of how object boundary is described, active contours can be classified into edge based [2]-[5], region based [6]-[8], and hybrid approaches [9]-[11]. For edge-based methods, it is assumed that object boundaries collocate with image intensity discontinuities which is widely adopted, for example, in depth estimation from stereo [12]. Region-based techniques, on the other hand, assume that object boundaries collocate with discontinuities in regional characteristics, such as color and texture. In other words, each object has its own distinctive and continuous regional features.

Region-based techniques have some obvious advantages over edge-based methods in that object boundary description based on image gradient can often be compromised by noise and weak edges. They are also less sensitive to initialization, while edgebased active contours are prone to local minima. Thus, it is often desirable for edge-based techniques to carefully place the initial contour. This assumes that the prior knowledge of the object location is available, which is not always true in reality. Existing techniques can only reduce this initialization dependency to a very limited extent. The balloon force [2] can only expand or shrink the contours. The bidirectionality of GVF can sometimes cause the contours to collapse on approach to the same boundary. Moreover, it has convergence issues caused by critical points. [1], [4], [13]. It is evidently clear that initialization invariance is particularly difficult to achieve for edge-based

Manuscript received August 17, 2008; revised August 25, 2009. First published September 22, 2009; current version published December 16, 2009. The associate editor coordinating the review of this manuscript and approving it for publication was Prof. Dan Schonfeld.

The author is with the Department of Computer Science, University of Swansea, Faraday Tower, Singleton Park, Swansea SA2 8PP, U.K. (e-mail: x.xie@swansea.ac.uk).

Color versions of one or more of the figures in this paper are available online at http://ieeexplore.ieee.org.

Digital Object Identifier 10.1109/TIP.2009.2032891 methods. More recent attempts, such as [4], [5], [13], and [14], showed promising but limited success [1].

In [1], Xie and Mirmehdi proposed a novel active contour model based on hypothesized magnetic interactions among gradient vectors and contours. This edge-based method showed significant improvements on convergence issues, e.g., reaching deep concavities. This paper extends the method so that the active contour can be randomly initialized and still be able to localize objects of interest. It can even perform segmentation without placing an initial contour. Fig. 1 gives an example of localizing two objects with internal holes and inhomogeneous intensity using an initial contour placed outside the objects. Only the proposed method successfully localized both external and internal boundaries. This initialization flexibility is particularly useful when prior knowledge of object location is not available and/or the initial contour is occluded from the object boundaries. We show in fact initial active contour is not necessary with the proposed method. This enhanced edge-based approach provides an effective alternative to region-based methods which may have difficulty in handling, for example, intensity inhomogeneity [e.g., see Fig. 1(d)].

\section{PRoposed Method}

\section{A. Basic MAC Model}

Fittings based on local intensity discontinuity can often lead to undesired local minima. The CPM [14] assigns opposite charges to edges and free particles so that the particles are pulled towards edges while repelling each other. This global interaction provides much freedom of initialization. However, particles on weak edges can be gradually pulled towards neighboring strong edges, resulting in broken boundaries [see Fig. 13(d)]. Particle addition and deletion and contour reconstruction can also be difficult in practice.

Instead of assigning fixed charges, we allow the charges flow through the edges. These flows of charges will then generate a magnetic field. The active contour, carrying similar flow of charges, will be attracted towards the edges under this magnetic influence. Without losing generality, let us consider the image plane as a 2-D plane in a 3-D space whose origin coincides with the origin of the image coordinates. Additionally, the third dimension of this 3-D space is considered perpendicular to the image plane.

The direction of the currents, flows of charges, running through object boundary can be estimated based on edge orientation, which can be conveniently obtained by a $90^{\circ}$ rotation in the image plane of the normalized gradient vectors $\left(\hat{I}_{x}, \hat{I}_{y}\right)$, 


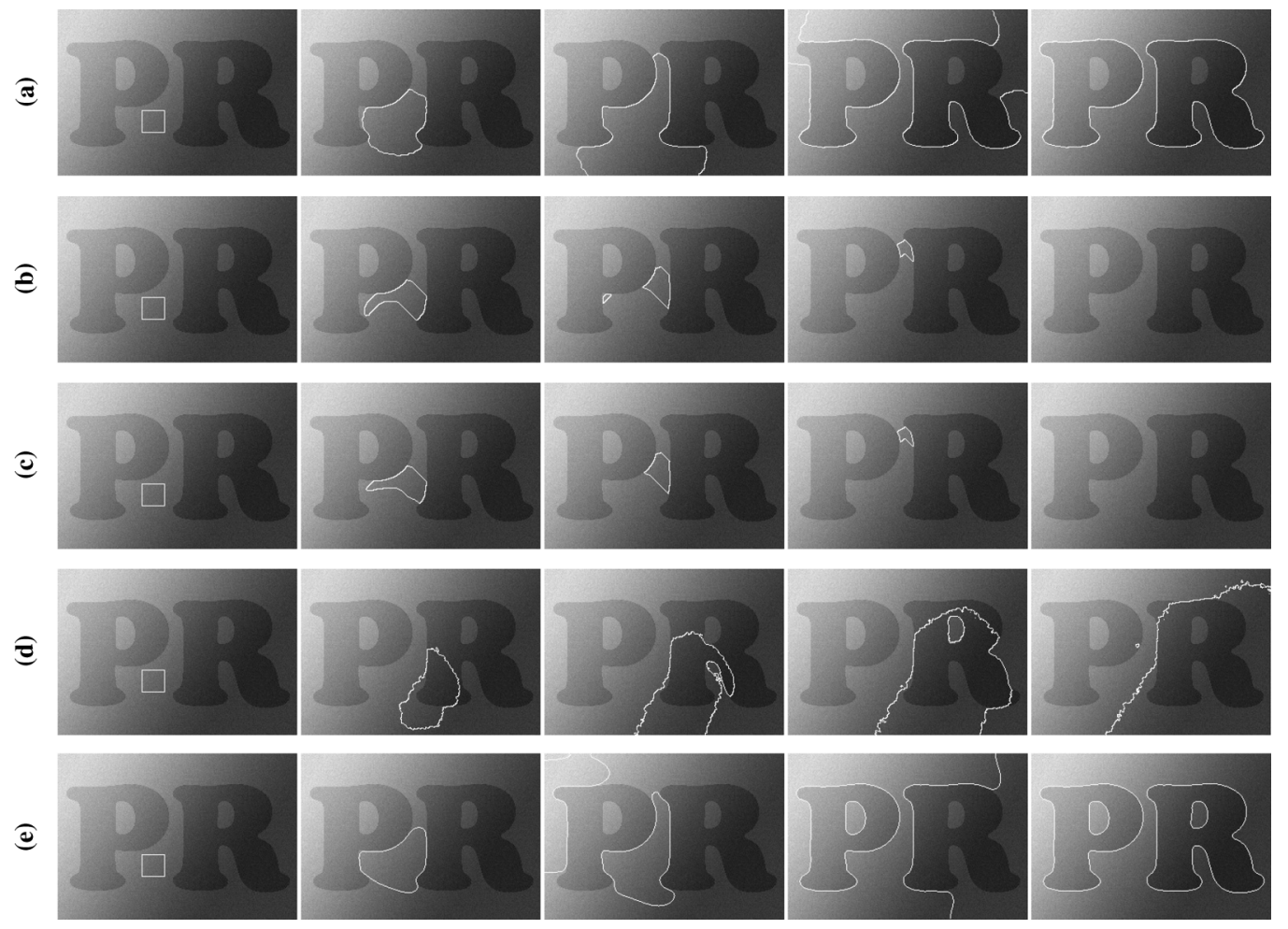

Fig. 1. Recovering objects with internal holes and inhomogeneous intensity. Row (a): Geodesic active contour [2]. ; Row (b): Generalized GVF (GGVF) active contour [3], [15]. Row (c): Geodesic GVF (GeoGVF) active contour [4]. Row (d): Chan-Vese model [6]. Row (e): Proposed method ( $K=0.02)$. Each row, from left to right, shows initial contour, intermediate stages, and stabilized result.

where $I$ denotes an image. Let $\mathbf{x}$ denote a point in the image domain. Thus, the object boundary current direction, $\mathbf{O}(\mathbf{x})$, can be estimated as: $\mathbf{O}(\mathbf{x})=(-1)^{\lambda}\left(-\hat{I}_{y}(\mathbf{x}), \hat{I}_{x}(\mathbf{x}), 0\right)$, where $\lambda=1$ gives an anti-clockwise rotation in the image coordinates, and $\lambda=2$ provides a clockwise rotation. However, we show later by using the proposed level set updating scheme different $\lambda$ values lead to the same result. Since the active contour is embedded in a signed distance function, the direction of current for the contour, denoted as $\Upsilon$, can be similarly obtained by rotating the gradient vector $\nabla \Phi$ of the level set function. Similar to $\mathbf{O}, \Upsilon$ is also 3-D and lies in the image domain, i.e.,

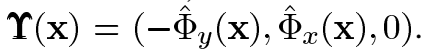

Let $f(\mathbf{x})$ be the magnitude of edge pixel and the magnitude of boundary current be proportional to edge strength, that is, the electric current on object boundary is defined as $f(\mathbf{x}) \mathbf{O}(\mathbf{x})$. The magnetic flux $\mathbf{B}(\mathbf{x})$ generated by gradient vectors at each pixel position $\mathbf{x}$ can then be computed as

$$
\mathbf{B}(\mathbf{x}) \propto \sum_{\mathbf{s} \in \mathbf{S}, \mathbf{s} \neq \mathbf{x}} f(\mathbf{s}) \mathbf{O}(\mathbf{s}) \times \frac{\hat{\mathbf{R}}_{\mathbf{x s}}}{R_{\mathbf{x s}}^{2}}
$$

where $\mathbf{s}$ denotes an edge pixel position, $\mathbf{S}$ is the set containing all the edge pixel positions across the image, $\hat{\mathbf{R}}_{\mathrm{xs}}$ denotes a 3-D unit vector from $\mathbf{x}$ to $\mathbf{s}$ in the image plane, and $R_{\mathrm{xs}}$ is the distance between them. Thresholding can be applied to remove some erroneous edge pixels with very small gradient magnitude
[1], [14]. The active contour is assigned with unit magnitude of electric current. The force imposed on it can be derived as

$$
\mathbf{F}(\mathbf{x}) \propto \boldsymbol{\Upsilon}(\mathbf{x}) \times \mathbf{B}(\mathbf{x}) .
$$

From (1) and (2), we can see that $\mathbf{B}$ intersects the image plane perpendicularly and $\mathbf{F}$ is always perpendicular to both $\boldsymbol{\Upsilon}$ and B. Thus, $\mathbf{F}$ also lies in the image domain and its third element equals to zero. For simplicity, from now on, we shall ignore its third dimensional component and denote $\mathbf{F}(\mathbf{x})$ as a 2-D vector field in the image domain. The basic model can then be formulated as

$$
C_{t}=\alpha g(\mathbf{x}) \kappa \hat{\mathbf{N}}+(1-\alpha)(\mathbf{F}(\mathbf{x}) \cdot \hat{\mathbf{N}}) \hat{\mathbf{N}}
$$

where $g(\mathbf{x})=1 /(1+f(\mathbf{x})), \kappa$ denotes the curvature, and $\hat{\mathbf{N}}$ is inward unit normal.

We can see from (1) and (2) that the image force is derived from global interactions among rotated gradient vectors, i.e., $f(\mathbf{x}) \mathbf{O}(\mathbf{x})$. Thus, it is more robust than fittings based on local gradient towards weak edges (where $f(\mathbf{x})$ is small) and noise (where $\mathbf{O}(\mathbf{x})$ is locally inconsistent). It is worth noting, however, that general contrast consistency along the object boundaries is important to the model. Large contrast variation can disrupt the force field, e.g., half of the object appears brighter than background and the other half appears to be darker. However, this does not mean that the entire object has to be brighter 
or darker than background. Those regions away from object boundary can be continuously varying in intensity. The model also can tolerate a fair amount of local contrast inconsistency, in the same way as to image noise and weak/broken edges.

\section{B. Level Set Regularization and Improved Initialization Independency}

As aforementioned, due to cross product computation the external force, $\mathbf{F}$, is always perpendicular to $\boldsymbol{\Upsilon}$ which is tangent to the contour, i.e., the external force is imposed along the normal direction. Note the internal force due to curvature flow is enforced in the inward normal direction. Thus, the total force is always perpendicular to active contour. In other words, it dynamically updates itself according to contour evolution to push and pull the contours along the normal direction until they reach object boundaries where forces from both sides are in balance. As a result, the propagating contour will not suffer from those convergence issues related to static force fields, such as GVF, in which evolving contours may become tangent to underlying force vectors resulting in false convergence. This force field is also significantly different from others used in edge-based methods. For example, in CPM, the force between an edge pixel $\mathbf{s}$ and an infinitesimal contour segment $\mathbf{c}$ lies in a straight line between these two, regardless the orientation of the contour segment. In our model, the orientation of the edge pixel and the contour segment also have influence on the resulting force interaction. This ability to adapt is very important since it ensures the active contour, once initialized, overcome deep concavities and narrow regions to reach object boundaries.

By incorporating (2), (3) can be re-written as

$$
\begin{aligned}
C_{t} & =\alpha g(\mathbf{x}) \kappa \hat{\mathbf{N}}+(1-\alpha)(\mathbf{\Upsilon}(\mathbf{x}) \times \mathbf{B}(\mathbf{x}) \cdot(\hat{\mathbf{N}}, 0)) \hat{\mathbf{N}} \\
& =\alpha g(\mathbf{x}) \kappa \hat{\mathbf{N}}+(1-\alpha)(\mathbf{B}(\mathbf{x}) \cdot((\hat{\mathbf{N}}, 0) \times \mathbf{\Upsilon}(\mathbf{x}))) \hat{\mathbf{N}}
\end{aligned}
$$

The external force in the second term is in fact a projection of the magnetic flux onto a binormal unit vector which is computed from a cross product of the contour inward normal and its tangent vector. A positive projection will force the contour to expand and a negative projection will shrink the contour, which acts in a similar way as what a region indication function does in a region-based approach, however, this is derived from the edge-based assumption. Thus, an edge can attract or push a contour which may lie either side of the edge. However, this bidirectionality is fundamentally different from that in, for example, GVF. In GVF, the force imposed on the contour is independent of the contour itself, which can cause the contours to collapse to each other when reaching to the same object boundary. For the proposed method, the force is related to both the image gradient and the contour (which can be clearly seen from (4)). It has the ability to prevent the contour from reaching to the same boundary and disappearing after merging together. This is illustrated in Fig. 2 with different initialization conditions and different topologies (see Fig. 2 for a detailed explanation of each scenario). This unique bidirectionality is very important in order to improve initialization independency. The active contour is allowed to be initialized across the object boundary without the

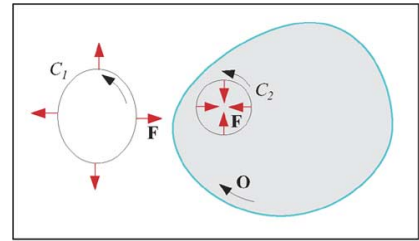

(A)

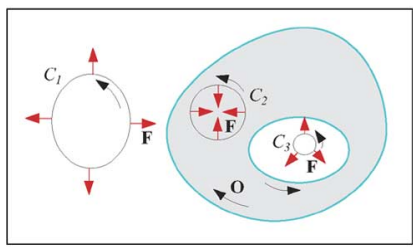

(C)

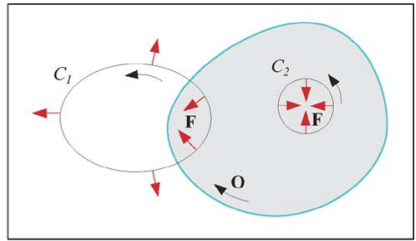

(B)

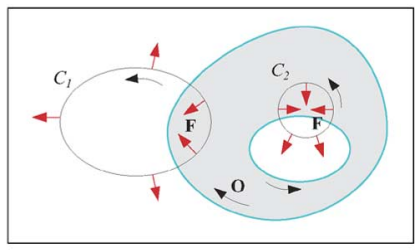

(D)
Fig. 2. Preventing contour collapsing. (a) Two contours, $C_{1}$ and $C_{2}$, are placed on each side of an object boundary with current directions indicated by arrows. Contour $C_{1}$ is attracted by the object boundary and expands itself in the outward normal direction. It eventually will wrap around and capture the object boundary. Contour $C_{2}$, however, is repelled and forced to shrink in the inward normal direction. Thus, two contours will not collapse to each other. (b) Similar to (a), however, contour $C_{1}$ is placed across the object boundary. Those contour segments of $C_{1}$ that are inside object boundary will be pulled towards object boundary and the rest of contour $C_{1}$ will expand and wrap around the object boundary. The segments inside object boundary and outside will not collapse to each other. (c) The object in this case contains an internal boundary. The behavior of $C_{1}$ and $C_{2}$ is similar to that in (a). Contour $C_{3}$ will expand itself to capture the internal boundary. Three contours will not collapse to each other, while capturing both boundaries. (d) Contours $C_{1}$ and $C_{2}$ are now initialized across external and internal boundaries, respectively. The behavior of $C_{1}$ is similar to that in (b). The contour segments of $C_{2}$ that are inside the object (gray area) will be attracted to the object internal boundary that is initially inside contour $C_{2}$. The other contour segments of $C_{2}$ will expand to capture the rest internal boundaries. No contour collapsing will occur, either. GVF contours, as an example, will collapse to each other in all above scenarios.

danger of collapsing to itself. Meanwhile, it still has the flexibility of splitting and merging. Contours or contour segments inside and outside object of interest freely merge together, while those in the vicinity of the object boundary are competing for edges. It thus allows random initialization where multiple contours can be arbitrary placed across the image (e.g., see Fig. 11).

The unique properties of this edge-based force interactions, i.e., dynamic force field and bidirectionality with anti-collapsing ability, provide a good foundation to improve initialization invariancy. However, in conventional level set methods, active contour is not able to create new components away from zero level set (existing contours) since periodic re-shaping the level set surface is necessary to maintain numerical stability. Ramlau and Ring [16] suggested to insert new contours once current contours were stabilized and examine the data fitting to determine whether to keep the new contour. However, this insertion and deletion can be difficult for complex images, particularly when segmenting small objects. Note the first term in (4) is effectively a weighted length minimization flow which can be achieved by minimizing this energy

$$
\mathcal{E}_{1}=\alpha \int_{\Omega} g(\mathbf{x})|\nabla \mathcal{H}(\Phi)| d \mathbf{x}
$$

where $\mathcal{H}($.$) is the Heaviside function. The second term in (4)$ can be viewed as an area minimization flow weighted by a triple 

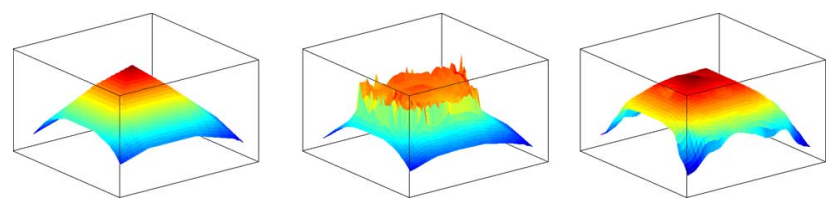

Fig. 3. From left: Initial level set surface, Chan-Vese level set surface after a few iterations, and level set surface using constrained level set updating after the same number of iterations.

product. Thus, the corresponding energy functional can be expressed as

$$
\begin{aligned}
\mathcal{E}_{2} & =(1-\alpha) \int_{\Omega}\left(\mathbf{B}(\mathbf{x}) \cdot\left(\frac{(\nabla \Phi, 0)}{|\nabla \Phi|} \times \mathbf{\Upsilon}(\mathbf{x})\right)\right) \mathcal{H}(\Phi) d \mathbf{x} \\
& =(1-\alpha) \int_{\Omega}(\mathbf{B}(\mathbf{x}) \cdot \mathbf{R}) \mathcal{H}(\Phi) d \mathbf{x}
\end{aligned}
$$

where $\mathbf{R}$ is a binormal unit vector, which is computed from the cross product of level set normal and its tangent vector, and it is perpendicular to the image plane. The active contour partition can then be carried out by minimizing the combined energy $\mathcal{E}=$ $\mathcal{E}_{1}+\mathcal{E}_{2}$. Minimization of this energy functional is possible via gradient descent using variational calculus

$$
\Phi_{t}=\alpha g(\mathbf{x}) \nabla \cdot\left(\frac{\nabla \Phi}{|\nabla \Phi|}\right) \delta(\Phi)-(1-\alpha)(\mathbf{B}(\mathbf{x}) \cdot \mathbf{R}) \delta(\Phi)
$$

where $\delta($.$) is the Dirac delta function. Following [6], we choose$ the regularized delta function as $\delta_{\epsilon}(z)=\epsilon /\left(\pi\left(\epsilon^{2}+z^{2}\right)\right)$, with $\epsilon=1$ so that its discretization has a support larger than zero which permits the perturbation away from zero level set. This updating scheme allows our active contour to create new contours away from existing ones, which gives great flexibility in initialization. To the best of our knowledge, there is no edgebased approach reported in the literature that has such degree of initialization independency.

However, also due to that delta function, $\delta_{\epsilon}($.$) , level sets close$ to zero level update much faster than others. This results in steep gradient around zero level set, which causes irregularities, destroys surface smoothness, and can slow down the contour evolution as more levels are pushed away from zero level set (see Fig. 3 as an example). An intuitive way to alleviate this problem is through constrained level set updating, that is, we empirically restrain the speed of level set updating for each iteration, i.e., $|\Phi(\mathbf{x}, t+1)-\Phi(\mathbf{x}, t)| \leq \tau$. From our experiments, we have found that using the level set time step as a guidance for the updating constraint, i.e., $\tau=\Delta t$, provides a good tradeoff between the degree of constrain and the speed of level set updating. It significantly reduces the risk of steep gradient. Fig. 3 shows an example of level set updating. Moreover, we propose to reduce the number of level set levels to a narrow band of zero level set, i.e., limit the range of the level set values by thresholding the level set to a narrow band. This significantly speeds up the convergence, particularly for creating new contours far away from existing ones.

This empirical approach, however, cannot guarantee the smoothness of the level set surface. Instead of implicitly imposing level set smoothing constraint, alternatively, we can explicitly smooth the level set surface to remove sharp

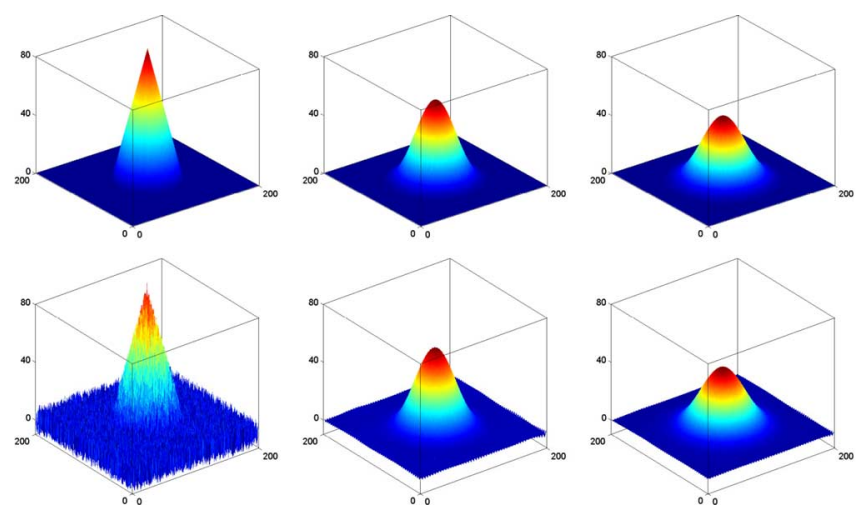

Fig. 4. First row: Steep level set surface and its evolution using proposed constrained level set diffusion method. Second row: Significant amount of independent noise is added to the level set function and its diffusion result using the proposed method. The figure in the right end of each row shows the stabilized diffusion result.

slopes based on anisotropic diffusion, which has been found very useful in, for example, edge detection [18]. Consider the following anisotropic diffusion equation:

$$
\Phi_{t}=\nabla \cdot(c(\Phi, t) \nabla \Phi)
$$

where $c($.$) denotes a diffusion function. Thus, \Phi$ is smoothed across level sets subject to the condition specified by $c$. Conventionally, level set function is initialized as a signed distance field, which has the characteristic of unit gradient magnitude [17], i.e., $|\nabla \Phi|=1$. Since we are only interested in reducing steep slopes and flat level set surfaces away from zero level are encouraged in order to develop new contours, the diffusion function can be specified as $c=\mathcal{H}(|\nabla \Phi|-1)$. To recap, due to the use of delta function, level sets near zero level are very unlikely developing any flat slopes. Thus, the constrained level set diffusion can be formulated as

$$
\Phi_{t}=\nabla \cdot(\mathcal{H}(|\nabla \Phi|-1) \nabla \Phi) .
$$

The diffusion is largely inactive when $|\nabla \Phi|<=1$ and actively takes place when $|\nabla \Phi|>1$. Steep surfaces are most likely occur near zero level set since these level sets updates much faster than those far away from zero level according to the delta function. Thus, this diffusion predominantly takes place around zero level set. It effectively minimize steep surfaces and also smoothes the zero level set, i.e., contour. The first row in Fig. 4 provides an example of constrained diffusion of a steep level set surface, i.e., $|\nabla \Phi|>1$. The proposed constrained diffusion effectively reduced level set gradient where necessary while maintaining its surface regularity. We also can see that when $|\nabla \Phi|>1$, (9) becomes the well studied heat equation with unit diffusivity. One of its well known properties is that any discontinuity will be quickly and effectively smoothed out. Thus, the proposed diffusion scheme can prevent the level sets around zero level from developing any irregularities and maintain their smoothness. The second row in Fig. 4 demonstrates the smoothing effect of this level set diffusion. A significant amount of noise was artificially introduced to a steep level set surface (as shown in the first row). The proposed method regularized the surface while reducing the level set steepness. Surface irregularities introduced 
by, for example, accumulated numerical errors due to local estimation of gradient using finite difference method or nonuniform surface propagation due to the use of delta function, can be effectively removed. This intrinsic regularization allows the level set function to evolve without reinitialization, which provides possibilities for more sophisticated topological changes, such as developing internal boundaries. It also allows the level set function to update more efficiently since the distortion by the delta function is constrained. The regularized Heaviside function $\mathcal{H}_{\epsilon}=(1 / 2)\left(1+(2 / \pi) \tan ^{-1}(z / \epsilon)\right)$, as introduced in [6], can be used so that it is coupled with the regularized delta function, i.e., $\mathcal{H}_{\epsilon}^{\prime}=\delta_{\epsilon}$, and a smooth transition between different level sets. It is worth noting that this regularized Heaviside function creates a much larger support. As a result, level sets with gradient magnitude less than one will also have some diffusion effect. However, this diffusion is very limited. A smaller $\epsilon$ value can be used, e.g., $\epsilon=0.5$, to reduce this effect. In practice, the regularized Heaviside function provides a smoother transition than a strict step function, which is beneficial for maintaining surface regularity.

As mentioned earlier, two different $\lambda$ values result in two opposite current directions on object boundary, i.e., $\mathbf{O}(\mathbf{x}, \lambda=$ $1)=-\mathbf{O}(\mathbf{x}, \lambda=2)$. Following (1) and (2), we then have $\mathbf{B}(\mathbf{x}, \lambda=1)=-\mathbf{B}(\mathbf{x}, \lambda=2)$, which further leads to $\mathbf{F}(\mathbf{x}, \lambda=$ $1)=-\mathbf{F}(\mathbf{x}, \lambda=2)$. In other words, the force is imposed in opposite direction along the normal for $\lambda=1$ and $\lambda=2$. So, as an example, if $\lambda=1$ expands the contour, $\lambda=2$ will shrink it. Since we are using level set, this means if $\lambda=1$ lifts up the level set values to positive then $\lambda=2$ will push down the level set values towards negative. Because the proposed method allows the level set to continuously evolve itself, the two level set functions eventually will be in the opposite phase. The zero level set, i.e., contour, however reaches the same place. Fig. 6 provides an example of level set evolution under different $\lambda$ values.

Notably, very recently in [17], the authors proposed a level set diffusion term in order to maintain the level set function close to the signed distance field, i.e., $\Phi_{t}=\nabla \cdot((1-(1 /|\nabla \Phi|)) \nabla \Phi)$. This can be considered as choosing the diffusion function $c=$ $1-(1 /|\nabla \Phi|)$. Diffusion takes place whenever $|\nabla \Phi| \neq 1$. Although this avoids re-initialization, it penalizes perturbations away from zero level set. It also creates unnecessary peaks and valleys in the regions away from zero level set in order to confine it to the signed distance function locally. For our purposes, diffusing level sets where $|\nabla \Phi|<1$ is not strictly necessary since we allow the level set surface to bend over to cross zero level in order to create new components, during which a decreasing of level set gradient for those regions is desirable. The risk of flat level set surface around zero level is very small due to the use of delta function. Thus, we can incorporate (9) into (7) to impose constrained level set diffusion. However, for simplicity and also due to the fact that this diffusion has the effect of contour smoothing, we replace the curvature term by this level set regularization term

$\Phi_{t}=\alpha \nabla \cdot\left(\mathcal{H}_{\epsilon}(|\nabla \Phi|-1) \nabla \Phi\right)-(1-\alpha)(\mathbf{B}(\mathbf{x}) \cdot \mathbf{R}) \delta_{\epsilon}(\Phi)$.

This constrained diffusion imposes inherent level set regularization, which ensures level set smoothness and prevents it from developing irregular surfaces, particularly, around zero level. The level set evolution becomes much more efficient. Moreover, it can handle more sophisticated topological changes than splitting and merging. It allows the active contour to develop new components, which is critical, for example, to detect internal boundaries. As a result, this makes it possible for the contours to reach those object boundaries that are occluded from the initial contour. This significantly improves its initialization independency.

Fig. 5 provides a comparative example of level set updating using different schemes. The underlying internal and external forces are identical. The level set is initialized in a way such that those levels outside a narrow band of zero level are flattened in order to speed up the convergence as discussed earlier. As shown in row (a), level set updating using the Chan-Vese method without regularization quickly developed very steep slopes and destroyed surface smoothness. The signed distance-based diffusion method [17], shown in row (b), produced numerous local peaks and valleys which was not desirable and could result in erroneous contours. The proposed conditionally constrained diffusion scheme effectively prevented the surface from developing steep slopes and maintained smoothness.

Thus, without the need of reconditioning the level set function, the underlying force field can continuously deform the level set surface. As a result, the proposed method has a great freedom in contour initialization, which is a significant improvement for edge-based methods. To summarize, the improvements on initialization independency are achieved due to the following three attributes of the proposed method.

1) The dynamic nature of the force field. The image force always perpendicularly imposes on the active contour and adapts itself according to contour evolution. This is mathematically convenient to prove. According to (2), force $\mathbf{F}$ is always perpendicular to tangent vector of the contour. Thus, the force is perpendicular to the contour and imposed along the normal direction, inwardly or outwardly. This ensures the contours to be pushed or pulled in their normal direction until they reach the object boundaries defined by the interactions among image gradients. The active contour will not suffer from those convergence issues related to static force field. It thus significantly helps to improve initialization independency.

Fig. 7 provides a comparative example of the GGVF field and the dynamic force field of the proposed method. The testing image consists multiple objects with three different types of topologies. The first row shows the initial active contour position and converged result using GGVF. The contour failed to reach deep concavity. A close examination of the vector field at the entrance of the concavity, as shown in the next two images, showed that once the evolving contour (indicated in white) became tangent to the underlying vectors it could not propagate further resulting in false convergence. Thus, for GVF or any other static force field, the initial contour needs to be carefully placed to avoid such issues. The second row gives the segmentation result using the basic MAC model with the same initialization. It successfully reached the deep concavity. The force field at the concavity entrance clearly show its 

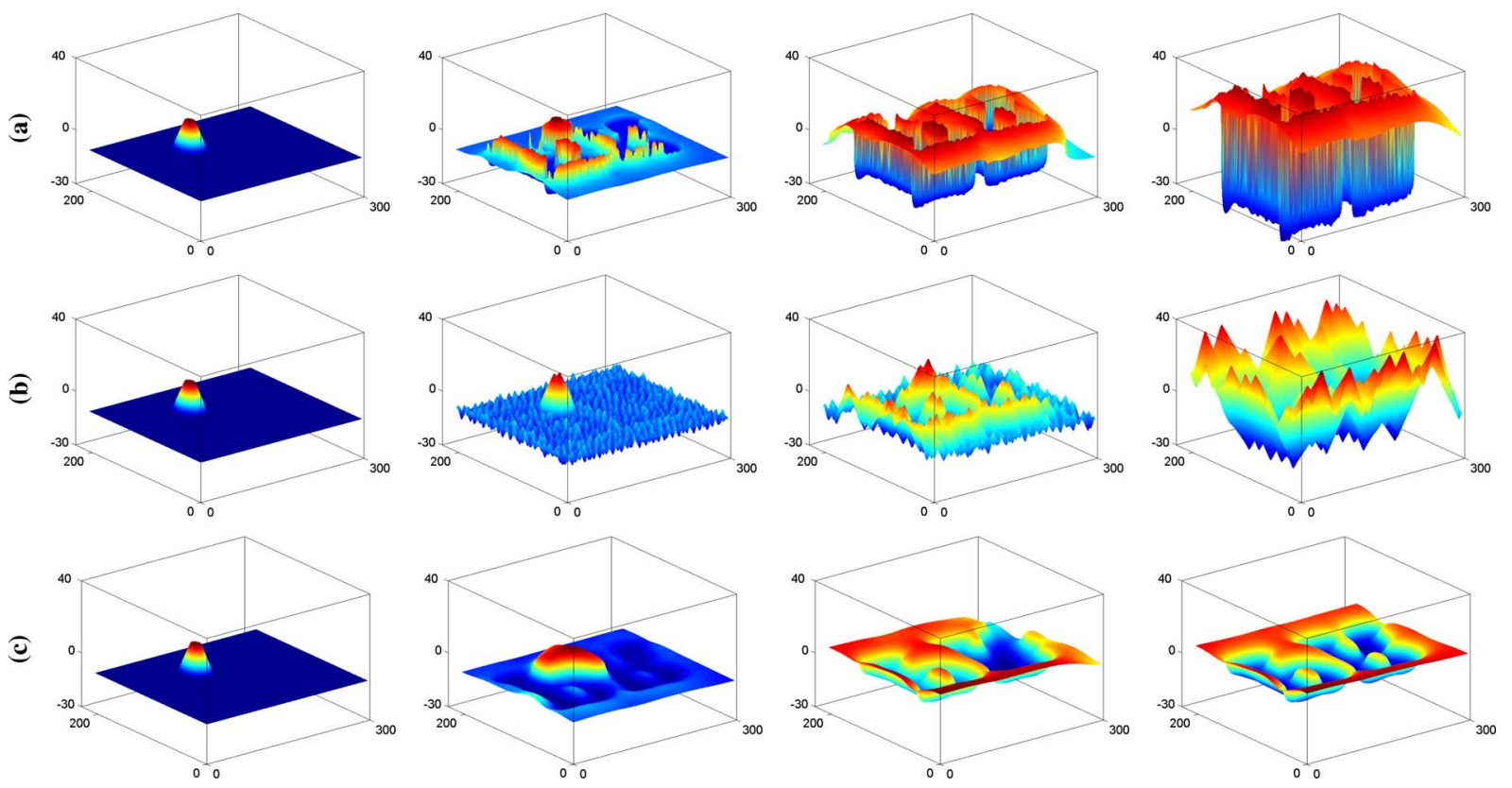

Fig. 5. Level set evolutions under the same internal and external forces but using different level set updating schemes. (a) Chan-Vese level set updating, (b) signed distance constrained diffusion [17], and (c) proposed constrained level set diffusion.
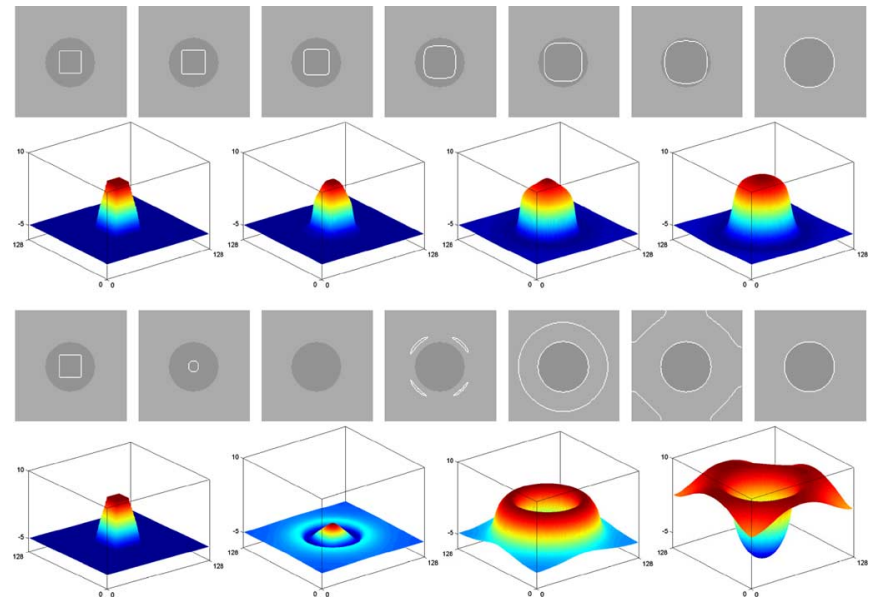

Fig. 6. Top two rows: contour and level set evolution when $\lambda=1$. Bottom two rows: contour and level set evolution when $\lambda=2$. The two level set evolved in opposite directions and resulted in opposite phase, with the zero level set reached the same place.

dynamic nature so that the forces are always perpendicularly imposed on the contour. The MAC model thus will not suffer from those convergence issues related to static force fields. However, although it provides great initialization flexibility, dynamic force field alone does not allow the active contour to capture all object boundaries, e.g., see Fig. 7.

2) Unique bidirectionality. The proposed active contour has a unique bidirectionality which prevents the contours from reaching the same object boundaries. This allows the contour to be initialized across object boundaries without the danger of collapsing to itself. Thus, the proposed method can handle random initializations, for example, as shown in Fig. 11. We can also verify this by examining (1) and (2). Suppose we have two infinitesimal contour segments, $C_{\mathbf{x}_{1}}$ and $C_{\mathbf{x}_{2}}$, which are nearest to an infinitesimal edge segment with edge orientation $\mathbf{O}(\mathbf{s})$, and one on each side of the edge. According to (1), we have $\mathbf{B}\left(C_{\mathbf{x}_{1}}\right) \propto f(\mathbf{s}) \mathbf{O}(\mathbf{s}) \times\left(\hat{\mathbf{R}}_{\mathbf{x}_{1} \mathbf{s}} / R_{\mathbf{x}_{1} \mathbf{s}}^{2}\right)$ and $\mathbf{B}\left(C_{\mathbf{x}_{2}}\right) \propto f(\mathbf{s}) \mathbf{O}(\mathbf{s}) \times\left(\hat{\mathbf{R}}_{\mathbf{x}_{2} \mathbf{s}} / R_{\mathbf{x}_{2} \mathbf{s}}^{2}\right)$. For convenience, we assume $R_{\mathbf{x}_{1} \mathbf{s}}=R_{\mathbf{x}_{2} \mathbf{s}}$ and take unit value for the constants. Since the contour segments are on opposite side of the edge and according to cross product computation, it is clear that $\mathbf{B}\left(C_{\mathbf{x}_{1}}\right)=-\mathbf{B}\left(C_{\mathbf{x}_{2}}\right)$. Thus, according to (2), the external force is imposed in the inward normal direction for one contour segment and outward normal direction for the other. This leads one contour to expand and capture the edge and the other to shrink and propagate away from the edge, which effectively prevents them from collapsing to the same edge segment.

This ability is further illustrated in Fig. 8, where multiple contours were initialized on both sides of an object boundary. The bidirectionality of GGVF resulted the contours collapsed to the same boundary, as shown in the first row. The unique bidirectionality of the MAC model, however, prevented them from collapsing to each other on object boundaries (see the second row). This property is essential when dealing with arbitrary number of initial contours. It is necessary in order to improve initialization independency. Note, however, this bidirectionality and dynamic force field are still not able to localize all the objects in the image.

3) Intrinsic level set regularization for advanced topological changes. The proposed constrained level set diffusion not only ensures surface regularity without reinitialization, but also allows more complex topological changes, such as developing internal boundaries and creating new components far away from existing contours. Using regularized delta 

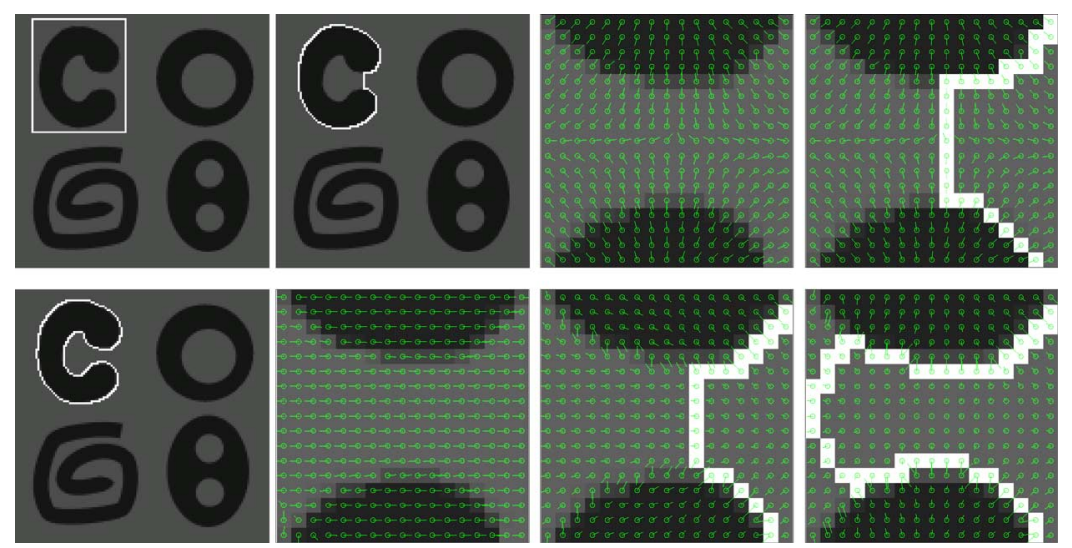

Fig. 7. First row, from left: Testing image with an initial GGVF contour, stabilized GGVF result, close-up of the vector field at the entrance of the concavity, and the final image shows the GGVF failed to propagate further once the contour (in white) was tangent to the underlying vectors. Second row, from left: Result of the basic MAC model, initial force field, adapting force field while the contour approaching the concavity, and the last image shows the dynamic force field continued to push the contour into the concavity to reach the object boundary.
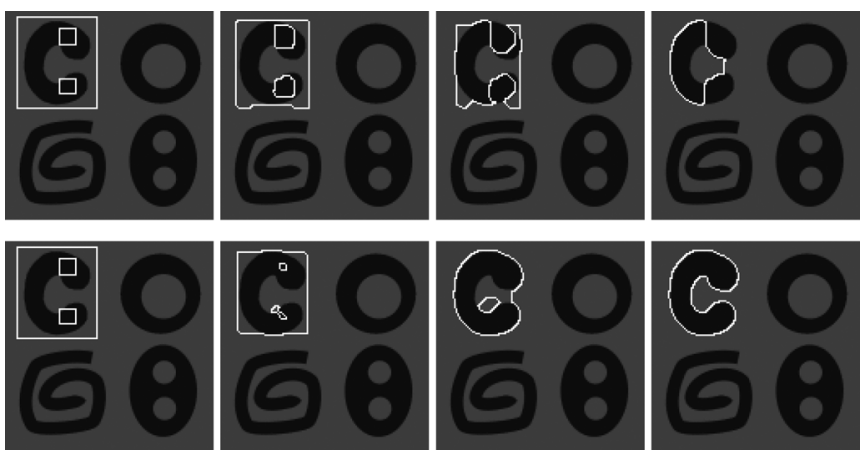

Fig. 8. Bidirectionality-Multiple contours were initialized on both sides of an object boundary. First row: GGVF contours collapsed to each other while reaching to the same object boundary from both sides. Second row: Unique bidirectionality of the proposed method successfully prevented contour collapsing.

function allows the evolution acts on all levels, not just locally, which also helps to achieve global minimum. Note, since the proposed method uses a single level set function, it is not possible to handle multiclass segmentation or multiple junctions. More than one level set functions is needed in order to handle this kind of topology [19].

In Fig. 9, the initial contour was placed inside one of the objects. Without the proposed level set evolution scheme, the MAC could not reach those object boundaries that were fully occluded from the initial contour (shown in the first row). The second row shows the proposed method successfully localized all the objects. The initial contour can be placed anywhere across the image, i.e., the placement of the initial contour does not affect the final segmentation result. We also show the result using a different $\lambda$ value in the third row, i.e., $\lambda=2$ instead of 1 . It produced different contour evolution, however, the same result.

The combination of these three properties gives the proposed active contour great flexibility in initialization and helps to achieve significant improvements in initialization independency. The nature of its force field serves as the foundation, whereas the level set updating scheme facilitates the necessary contour evolution. In fact, we show later that the proposed
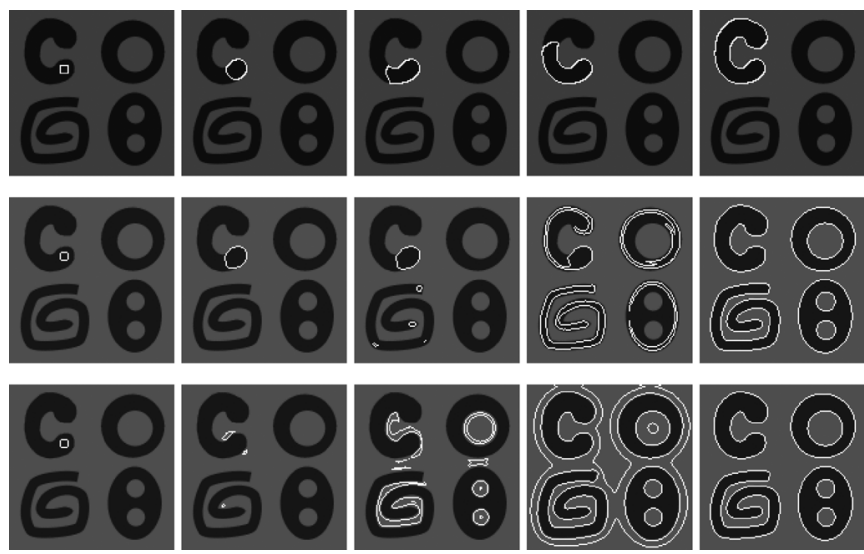

Fig. 9. Initial contour was placed inside one of the four objects. First row: Basic MAC model failed to localize other objects which were occluded from the initial contour. Second row: Proposed level set updating scheme helped the MAC to localize all the objects $(\lambda=1)$. Third row: Proposed method with $\lambda=2$.

method can be completely initialization-free, i.e., no initial contour. To the best our knowledge, this degree of initialization independency for edge-based model has not been reported before.

\section{Anisotropic Force Diffusion}

In [1], we proposed to perform nonlinear diffusion of the magnetic field in order to overcome noise interference when necessary. Here, we generally follow this approach but also add an edge saliency measure to the weighting function in order to better preserve the edges. Let $B(\mathbf{x})$ denote the signed magnitude of $\mathbf{B}(\mathbf{x})$. The diffused field $\hat{\mathcal{B}}(\mathbf{x})$ is obtained by solving

$$
\mathcal{B}_{t}(\mathbf{x})=p(B(\mathbf{x})) \nabla^{2} \mathcal{B}(\mathbf{x})-q(B(\mathbf{x}))(\mathcal{B}(\mathbf{x})-B(\mathbf{x}))
$$

where $p(B(\mathbf{x}))=e^{-(|B(\mathbf{x})| \mathcal{S}(\mathbf{x}) / K)}, q()=.1-p($.$) , and$ $\mathcal{S}($.$) is an edge saliency measure which is measured based on$ edge strength and orientation coherency, i.e., $\mathcal{S}(\mathbf{x})=f(\mathbf{x}) v(\mathbf{x})$ where $v($.$) is the variance of orientation in a local neighborhood,$ e.g., $9 \times 9$ as used here. More sophisticated saliency measures, e.g., [20], can be used. Weighting the flux magnitude with $\mathcal{S}($.) 


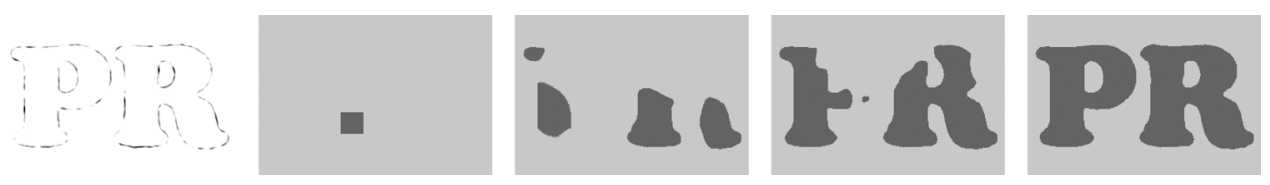

Fig. 10. Recovering broken edges—From left: Edge map which has significant amount of weak and broken edges, initial shape, intermediate stages, and recovered shape (stabilized result) using the proposed method $(K=0)$.

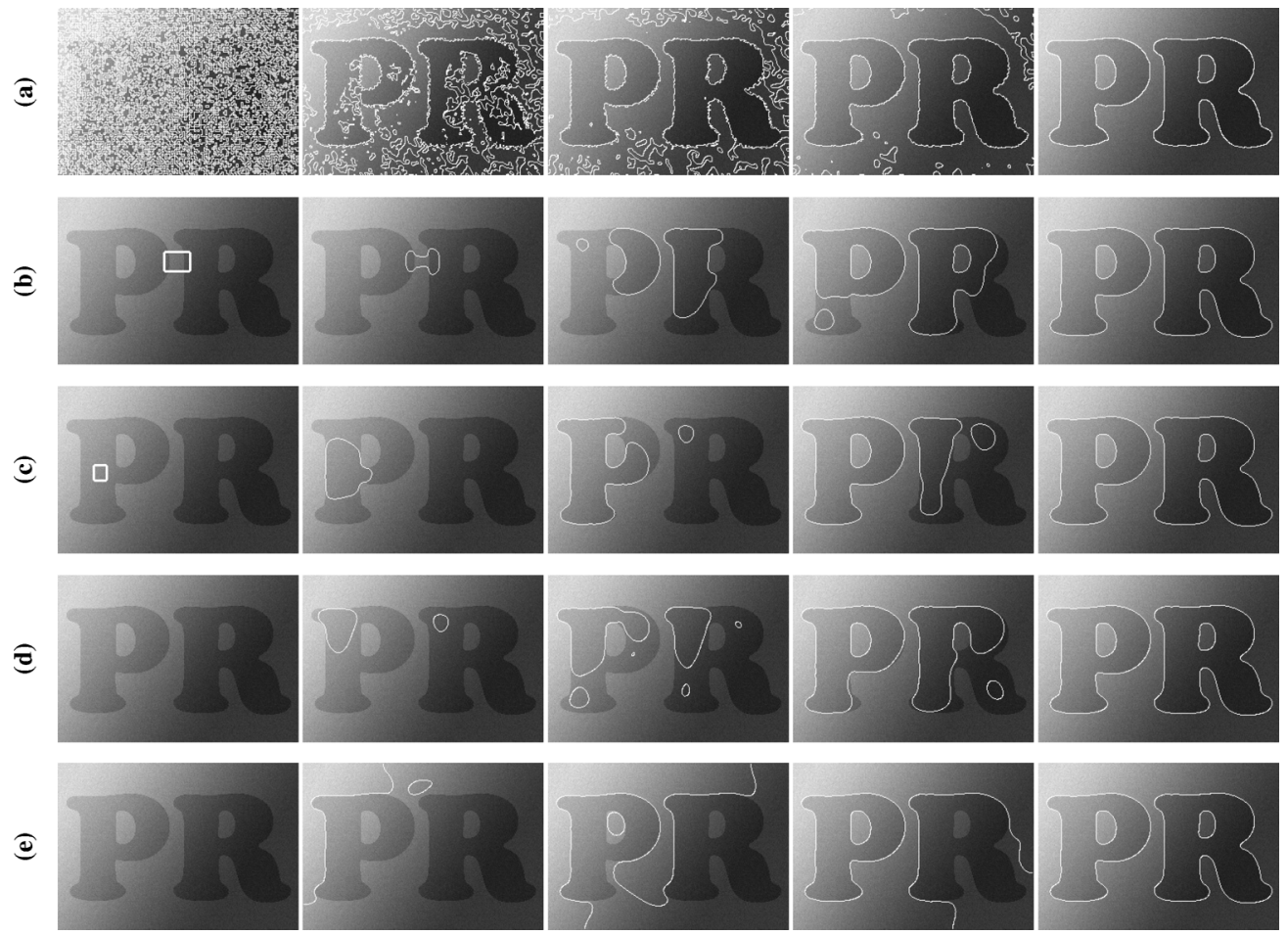

Fig. 11. Initialization flexibility—Row (a): Proposed method with numerous initial contours randomly placed across the image domain. Row (b): Cross boundary initialization. Row (c): Single initial contour placed inside one of the two objects; the other object is occluded from the initial contour. Row (d): Proposed method without any initial contour $(\lambda=1)$. Row (e): Proposed method without any initial active contour $(\lambda=2) . K=0.02$ for all cases.

further ensures as little diffusion as possible at object boundaries, while areas lack of consistent support from edges result in substantial diffusion.

\section{EXPERIMENTAL RESULTS}

The parameters involved are: $\alpha, \lambda$, and $K$. The value of $\alpha$ is fixed to 0.25 and $\lambda$ can be disregarded as a parameter since both values lead to the same result. The diffusion parameter $K$ should be used when noise interference is significant. We provide the value of $K$ for each example. Due to the intrinsic level set regularization, the time step for level set updating can be much larger than that in a conventional method. Following [17], we tested a wide range of time steps and similarly found that $\alpha \Delta t<0.25$ gave us very stable results.

The proposed method has been tested on a variety of synthetic and real images. In Fig. 1, to briefly recap, five methods were tested on a synthetic image containing two objects with internal holes. Only the proposed method could recover both the outer and inner boundaries. The ability to localize internal boundaries is very important in order to improve initialization invariancy. It also gave an example where the piecewise constant assumption is not sufficient due to inhomogeneity but the edge-based assumption could still be valid.
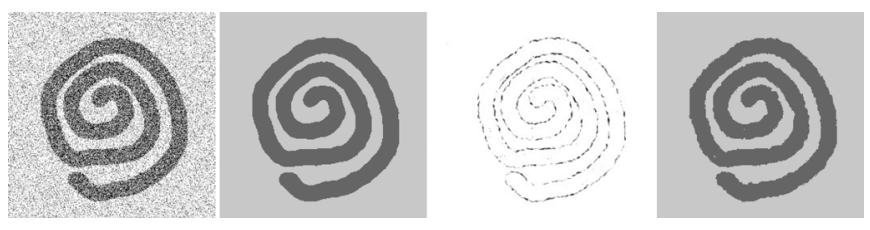

Fig. 12. From left: Complex shape with $50 \%$ Gaussian noise and the recovery result using force diffusion ( $K=0.2$ ); heavily thresholded edge map with numerous broken and weak edges and its recovery result without force diffusion, i.e., $K=0$.

However, edge-based assumption can often be compromised by weak edges, where intensity discontinuity is small but still can be important in localizing the object. In Fig. 10, we artificially create weak and even broken edges by heavily thresholding the edge map. The proposed method still managed to recover the objects without any dedicated initializations.

Fig. 11 further demonstrates such initialization flexibility. In row (a), numerous initial contours were randomly placed. The unique bidirectionality prevented the contours from collapsing to each other on object boundaries. A cross boundary initialization was shown in row (b). The contour did not collapse to itself, but split and converged correctly. In row (c), a single initial contour was placed inside one of the two objects. Despite the occlusion, the proposed method still localized both objects. 


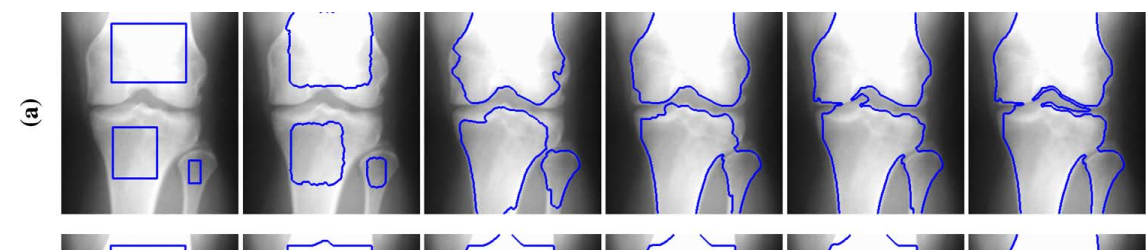

a

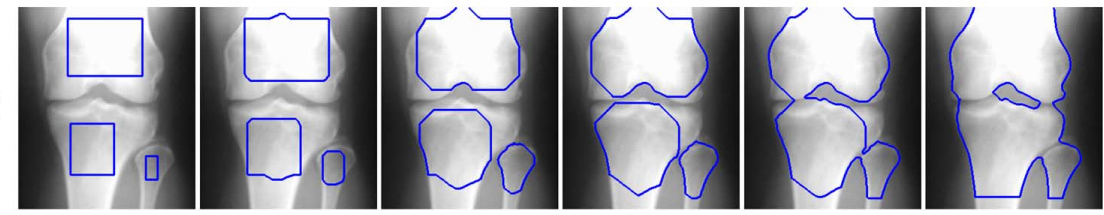

e

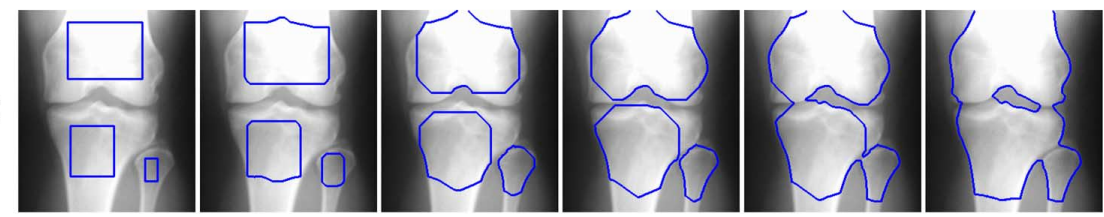

อิ

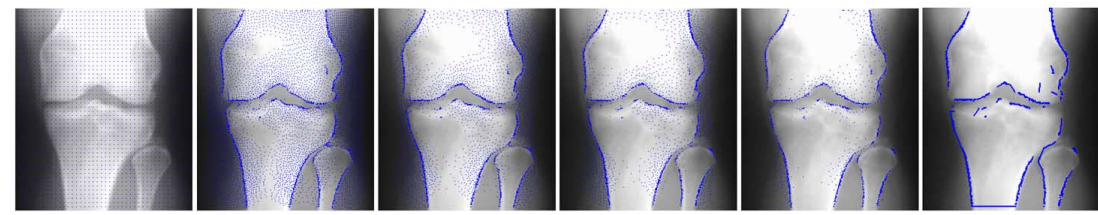

(2)

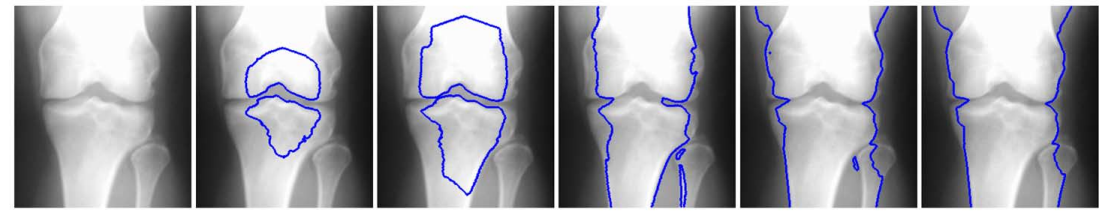

$\Theta$

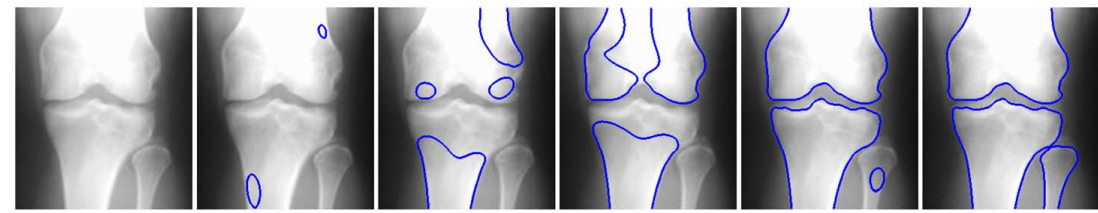

5

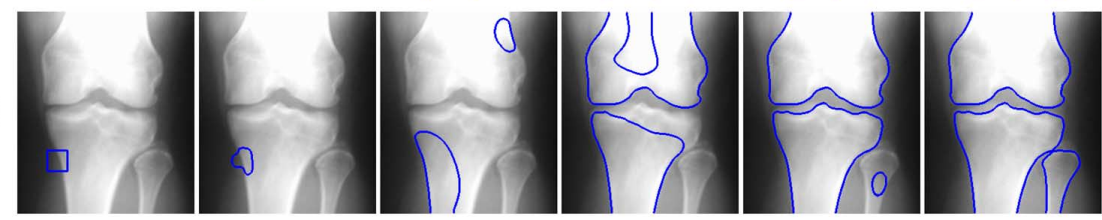

$\widehat{\varepsilon}$

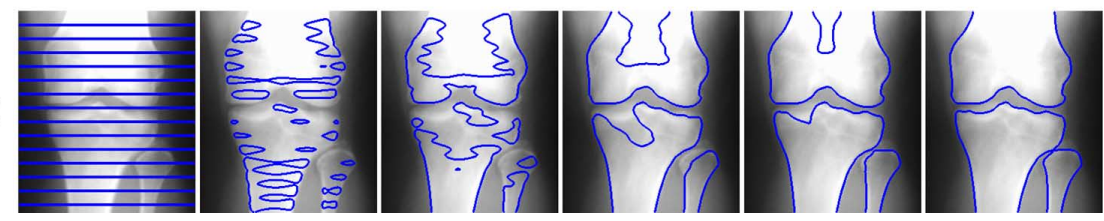

Fig. 13. Segmentation comparison. Row (a): Geodesic; row (b): GGVF; row (c): GeoGVF; row (d): CPM [14]; row (e): Chan-Vese model; row (f): proposed method without any initial active contour $(K=0.03)$; row $(\mathrm{g})$ : proposed method with a cross boundary initialization; row (h): proposed method with horizontal lines as initial contours. Note the initial contours or particles for all other edge-based methods were carefully placed in order to achieve best results.

In row (d), the initial level set was generated in a way such that there was no zero level, i.e., without any initial active contour. New contours were still able to emerge and correctly converged to the boundaries. In row (e), the same initialization was used but with opposite edge orientation, i.e., $\lambda=2$ instead of 1 . This resulted in a different contour evolution, however, the result was identical. For example, if $\lambda=1$ expands the contour, $\lambda=2$ would shrink the same contour at the same position. In conventional level set updating, this would be a problem since the user has to choose an appropriate $\lambda$ value beforehand. However, with the proposed level set updating scheme, the force field can continuously deform the level set function without the need of re-conditioning so that new contours will emerge to localize the objects.

Fig. 12 shows a complex shape with 50\% noise corruption. In the first row, the force diffusion was applied and achieved good result. In the second row, we performed heavy edge thresholding, which removed most noise edges but also produced numerous broken and weak edges. The proposed method still managed to recover its shape. This demonstrates the flexibility of the proposed method in dealing with image noise. However, it is worth noting that this is after all an edge-based method. 

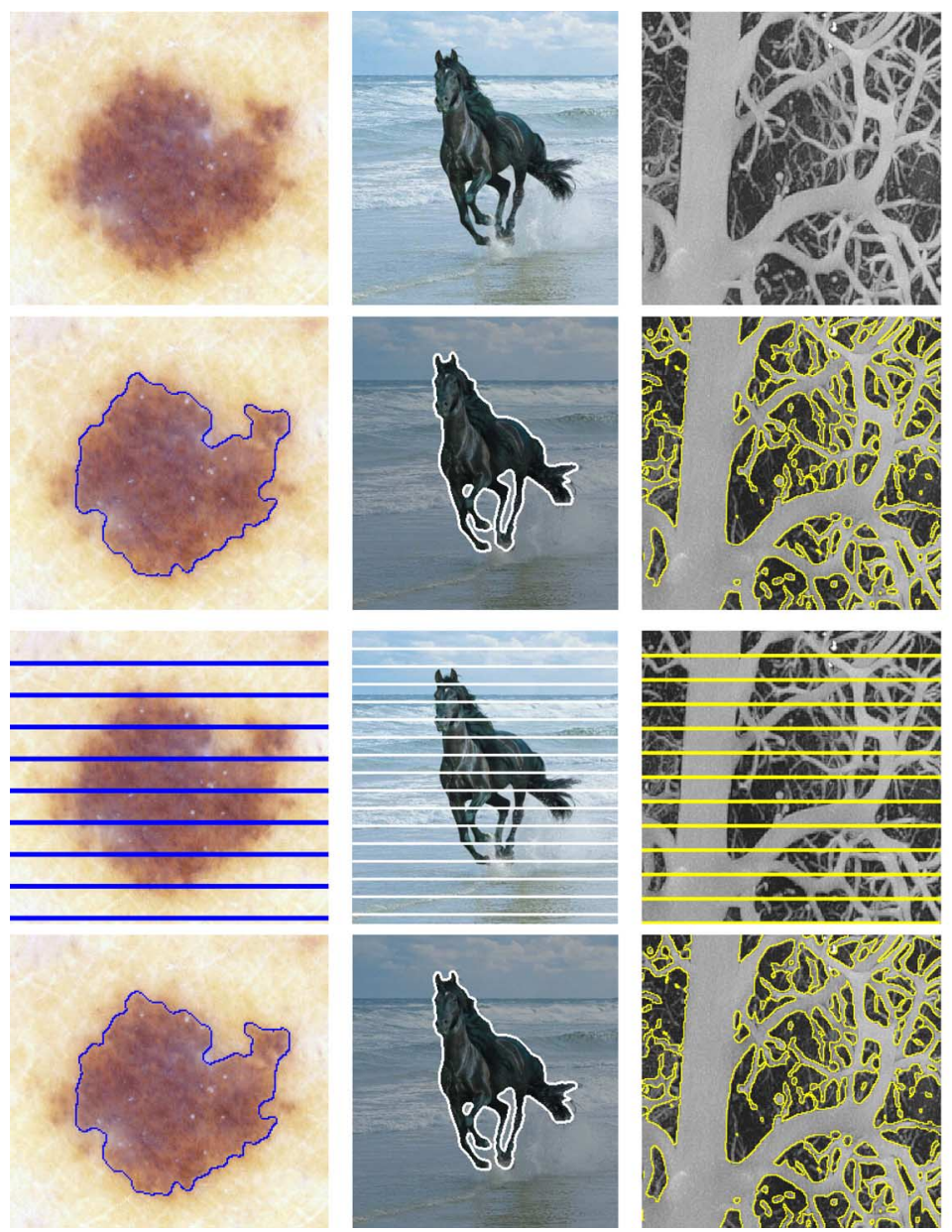
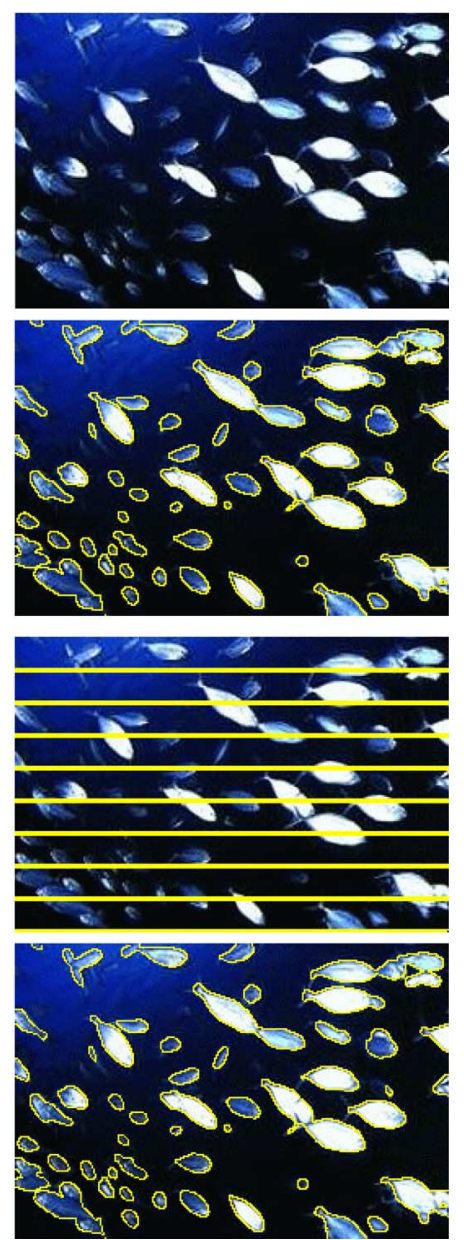

Fig. 14. Segmentation without initial active contour (color images). Top two rows-Segmenting the images without any initial active contour. From left: Skin lesion image which contains weak edges and image noise $(K=0.2)$; a horse image with large color variations and weak edges $(K=0.2)$; a blood vessel image with very complex shape and topology, varying intensity, and weak edges $(K=0)$; a fish swarm image with inhomogeneous color distribution, complex topology, and weak edges $(K=0)$. Bottom two rows-Segmenting the images using horizontal lines as initial contours (with the same parameter settings as used in the top two rows). Note the result of the horse image is dimmed in order to better visualize the contours.

Overwhelming noise interference will inevitably degrade its performance.

Fig. 13 gives comparative results on a CT bone scan. Note, for methods we compared against, the initial contours were carefully placed in order to achieve best possible results. However, due to excessive amount of weak edges, none of those methods succeeded. The geodesic active contour and CPM method failed on weak edges. Besides suffering from the convergence issue, the GGVF active contour failed in neighboring weak edge and strong edge and contours collapsed to each other. The GeoGVF active contour showed very limited improvement. The surrounding soft tissue caused the bone area and the rest regions statistically inseparable, which led the Chan-Vese model to over-segment the bone regions. The proposed method achieved good result even without any initial active contour [row (f)]. In row $(\mathrm{g})$, the result of a cross boundary initialization is also given. The initial contour was occluded from one of the objects to be segmented. We further tested the stability of the proposed method by using horizontal lines as initial contours, see row (h). There was no discernable difference between the results obtained using these three completely different initialization methods.
Examples of the proposed method on color images are given in Fig. 14. The lesion image contains significant amount of diffused edges. In the second example, there are large color variations in the horse body region, as well as weak edges. The blood vessel image is extremely challenging for edge-based active contour methods, since it contains numerous thin, complex structures, which makes it practically impossible for other edgebased methods, such as GVF. There are also intensity variations and plenty of weak edges. The proposed method not only localized distinctive structures with relatively large intensity contrast but also complex, detailed structures with much less contrast. The final example again demonstrates the initialization flexibility of the proposed method. The image contains a large number of objects with inhomogeneous color distributions and weak edges. The proposed method produced very good results in all cases, even without any initial contour (top two rows). Additional results using horizontal line initialization are also provided in Fig. 14 (bottom two rows). This again demonstrates the robustness of the proposed method. Edge detection in color images was done by following the method in [21]. 


\section{CONCLUSION}

We presented an edge-based active contour model, which showed superior performance, particularly, in initialization flexibility, as well as in handling weak/broken edges and inhomogeneity. Its novel level set diffusion allows the active contour efficiently update itself and detect object boundaries that are initially occluded. It is worth noting that its performance relates to the consistency of edge orientation which can be compromised by excessive amount of noise and busy texture. However, it does tolerate a fair amount of inconsistency since it takes into account interactions among gradient vectors, with the additional help from edge preserved diffusion. Its initialization flexibility opens new potentials for edge-based methods. It can be very useful, for example, in automatic object detection and localization.

\section{REFERENCES}

[1] X. Xie and M. Mirmehdi, "MAC: Magnetostatic active contour," IEEE Trans. Pattern Anal. Mach. Intell., vol. 30, no. 4, pp. 632-646, Apr. 2008.

[2] V. Caselles, R. Kimmel, and G. Sapiro, "Geodesic active contour," Int. J. Comput. Vis., vol. 22, no. 1, pp. 61-79, 1997.

[3] C. Xu and J. Prince, "Snakes, shapes, \& gradient vector flow," IEEE Trans. Image Process., vol. 7, no. 3, pp. 359-369, Mar. 1998.

[4] N. Paragios, O. Mellina-Gottardo, and V. Ramesh, "Gradient vector flow geometric active contours," IEEE Trans. Pattern Anal. Mach. Intell., vol. 26, no. 3, pp. 402-407, Mar. 2004.

[5] C. Li, J. Liu, and M. Fox, "Segmentation of edge preserving gradient vector flow: An approach toward automatically initializing and splitting of snakes," Comput. Vis. Pattern Recognit., pp. 162-167, 2005.

[6] T. Chan and L. Vese, "Active contours without edges," IEEE Trans. Image Prcoess., vol. 10, no. 2, pp. 266-277, Feb. 2001.

[7] N. Paragios and R. Deriche, "Geodesic active regions and level set methods for supervised texture segmentation," Int. J. Comput. Vis., vol. 46, no. 3, pp. 223-247, 2002.

[8] D. Cremers, M. Rousson, and R. Deriche, "A review of statistical approaches to level set segmentation: Integrating color, texture, motion and shape," Int. J. Comput. Vis., vol. 72, no. 2, pp. 195-215, 2007.

[9] J. Haddon and J. Boyce, "Image segmentation by unifying region and boundary information," IEEE Trans. Pattern Anal. Mach. Intell., vol. 12, pp. 929-948, 1990.
[10] A. Chakraborty, H. Staib, and J. Duncan, "Deformable boundary finding in medical images by integrating gradient and region information," IEEE Trans. Med. Imag., vol. 15, no. 6, pp. 859-870, Jun. 1996.

[11] X. Xie and M. Mirmehdi, "RAGS: Region-aided geometric snake," IEEE Trans. Image Process., vol. 13, no. 5, pp. 640-652, May 2004.

[12] S. Birchfield and C. Tomasi, "Depth discontinuities by pixel-to-pixel stereo," Int. J. Comput. Vis., vol. 35, no. 3, pp. 269-293, 1999.

[13] D. Gil and P. Radeva, "Curvature vector flow to assure convergent deformable models for shape modelling," in Proc. EMMCVPR, 2003, pp. 357-372.

[14] A. Jalba, M. Wilkinson, and J. Roerdink, "CPM: A deformable model for shape recovery and segmentation based on charged particles," IEEE Trans. Pattern Anal. Mach. Intell., vol. 26, no. 10, pp. 1320-1335, Oct. 2004.

[15] C. Xu and J. Prince, "Generalized gradient vector flow external forces for active contours," Signal Process., vol. 71, no. 2, pp. 131-139, 1998.

[16] R. Ramlau and W. Ring, "A Mumford-Shah level-set approach for the inversion and segmentation of X-ray tomography data," J. Comput. Phys., vol. 221, pp. 539-557, 2007.

[17] C. Li, C. Xu, C. Gui, and M. Fox, "Level set evolution without re-initialization: A new variational formulation," Comput. Vis. Pattern Recognit., pp. 430-436, 2005.

[18] P. Perona and J. Malik, "Scale-space and edge detection using anisotropic diffusion," IEEE Trans. Pattern Anal. Mach. Intell., vol. 12, no. 7, pp. 629-639, Jul. 1990.

[19] L. Vese and T. Chan, "A multiphase level set framework for image segmentation using the Mumford and Shah model," Int. J. Comput. Vis., vol. 50, pp. 271-293, 2002.

[20] G. Heidemann, "The long-range saliency of edge- and corner-based saliency points," IEEE Trans. Image Process., vol. 14, no. 11, pp. 1701-1706, Nov. 2005.

[21] J. Scharcanski and A. Venetsanopoulos, "Edge detection of color images using directional operators," IEEE Trans. Circuits Syst. Video Technol., vol. 7, no. 2, pp. 397-401, Feb. 1997.

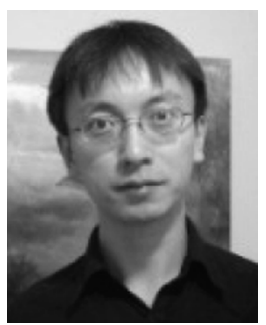

Xianghua Xie (M'02) received the M.Sc. (with commendation) and Ph.D. degrees in computer science from the University of Bristol, U.K., in 2002 and 2006, respectively.

Since 2007, he has been a Lecturer (RCUK Academic Fellow) in the Department of Computer Science, University of Swansea. He was previously a research associate in the Department of Computer Science, University of Bristol. His current research interests are video analysis, texture analysis, image segmentation, surface inspection, deformable models, and medical image analysis.

Dr. Xie is a member of the BMVA. 